\title{
Protée
}

\section{Vestige, archive et trace : Présences du temps passé}

\section{Herman Parret}

Volume 32, numéro 2, automne 2004

L’archivage numérique : conditions, enjeux, effets

URI : https://id.erudit.org/iderudit/011171ar

DOI : https://doi.org/10.7202/011171ar

Aller au sommaire du numéro

Éditeur(s)

Département des arts et lettres - Université du Québec à Chicoutimi

ISSN

0300-3523 (imprimé)

1708-2307 (numérique)

Découvrir la revue

Citer cet article

Parret, H. (2004). Vestige, archive et trace : Présences du temps passé. Protée, 32(2), 37-46. https://doi.org/10.7202/011171ar

\section{Résumé de l'article}

Ce texte constitue une préface philosophique à la pronominalisation technique et locale concernant la numérisation de l'héritage culturel. Quelques inquiétudes sont soulevées. Psychologiquement et phénoménologiquement, la numérisation peut-elle intégrer l'immanence corporelle de la mémoire, la variété des modes mnésiques et surtout la force vive de l'oubli dans la constitution de la mémoire ? Sémiotiquement, la numérisation peut-elle sauvegarder, voire cultiver, la trace et l'archive - lieux de la mémoire -, ce réel entre le possible (le schéma, la déduction, l'algorithme) et le réalisé (le positif, l'empirique, l'histoire et les discours)? 


\title{
VESTIGE, ARCHIVE ET TRACE PRÉSENCES DU TEMPS PASSÉ
}

\author{
HERMAN PARRET
}

TROIS FAÇONS D'HÉRITER LA CULTURE

Il semble bien qu'à toute époque les hommes et leurs sociétés ont eu conscience d'hériter la culture des ancêtres, ou des civilisations antérieures, et ont senti l'obligation de transmettre leur culture aux générations futures. Que la présence de cet héritage soit mythiquement projetée ou historiquement établie, comme dans la société moderne et scientifique, repose, semble-t-il, sur cette inclination profondément existentielle, collective et universelle des êtres humains à faire durer, à éterniser les cultures, à conserver reliques, monuments, vestiges et archives, toute la gamme des traces et des indices du passé qui procurent à notre vie l'horizon d'une histoire, la profondeur d'une origine. Peu de civilisations échappent à la manie patrimoniale, et certainement pas la nôtre. On a pu parler de l'esprit de muséalisation de notre temps. On assiste, il est vrai, à la muséalisation généralisée de l'existence, même actuelle, à cet effort global de récupération «muséale» des brins les plus intimes de notre expérience, à l'archivation d'infimes portions d'information.

Comment les cultures du passé sont-elles présentes dans la conscience et dans les pratiques des «héritiers» que nous sommes? Une taxinomie facile et trop simple fait la distinction entre la culture indiciaire, la culture textuelle et la culture mnésique. L'épistémologie que je formulerai dans la seconde partie de cet article s'efforcera de démontrer que ces trois «natures» de la culture et leur qualification sémio-épistémologique ne sont pas exclusives, mais s'interdéfinissent. Ce n'est que par méthode que je propose, en guise d'introduction, une taxinomie à trois termes. La culture indiciaire réside dans des vestiges-indices analysés par l'archéologie et témoigne des civilisations passées. La culture textuelle rassemble tous les «textes» produits par la créativité humaine, qu'ils soient artistiques ou simplement instrumentaux. Cette culture textuelle est surtout présente dans les bibliothèques, les archives et les musées. La culture mnésique est investie dans la mémoire des individus et des collectivités. Cette mémoire peut être codée et matérialisée dans les styles de vie, les habitudes sociétales, même dans l'urbanisme des villes. On pourrait caractériser sémiotiquement ces trois natures de la culture à l'aide des notions de vestige, d'archive et de trace. Les vestiges sont des indices dont le 
sens ne peut être reconstruit que par et dans la diachronie d'une entropie. Les archives sont des textes synchroniquement autonomes, fonctionnant comme des marques objectivées d'une subjectivité cogitante et désirante. Les traces sont les empreintes mentales que l'on ne peut penser adéquatement que sur le modèle de l'incision. Il s'agit évidemment des souvenirs et des anamnèses, d'une "mémoire" collective et individuelle, non écrite, spontanée et pragmatique. Nous, «héritiers» des cultures antérieures (mais non pas radicalement révolues), sommes interpellés par cette triple présence du temps passé et fascinés par l'identification des vestiges, des archives et des traces des cultures indiciaires, textuelles et mnésiques: identification par le retour archéologique aux origines d'avant l'entropie, par l'enchâssement dans une subjectivité productrice, par le contrôle véridictoire des souvenirs et des remémorations.

Les textes de ce dossier de Protée ne remettront sans doute pas en cause cette conception de l' héritage culturel, mais certains poseront, peut-être avec inquiétude, la question de savoir si l'ère des nouvelles technologies électroniques change radicalement les expectations à l'égard du maintien et de la transmission de l'héritage culturel. L'infrastructure numérique, pour certains, perturbe le processus traditionnel de sauvegarde et de transfert des produits culturels du passé. Bouleversement chez les uns, enthousiasme chez d'autres, nulle part indifférence. Le slogan d'Ivan Illich, "Un bulldozer se cache dans tout ordinateur», nous rend sensible, au moins, au fait que

[...] les nouveaux médias restent [...] à même de fixer et de transmettre des informations, mais ils ajoutent à ces deux fonctions classiques une troisième fonction, celle de la manipulation du savoir et de l'information, laquelle paraît se mettre en place au détriment des deux autres.

Pire, «l'héritage culturel est pris en charge par une infrastructure digitale, qui à la fois le rend plus aisément accessible et le détruit subrepticement» (Illich, 1991: 41). On a souvent commenté, voire déploré, les effets de l'archivage numérique de l'héritage culturel, par une triple prédication: pétrification, dématérialisation, visualisation du sens. Pétrification désigne la perte du vivant, de l'existentiel, du subjectal, du psychologique dans sa richesse et sa variation; dématérialisation signifie décontextualisation, abstraction, dissociation du «mental» d'une part et du corporel et du physique de l'autre; et visualisation, c'est-à-dire scénographie, cartographie, mise en images du cognitif et du pathétique, architectonique théâtrale sans temporalité, tout synchronique. Il ne convient pas de dramatiser le «changement paradigmatique»là où on passe du scriptural à la numérisation - ni de le minimiser, et certainement pas de trouver des réponses à des questions, mais tout simplement d'esquisser, en épistémologue, les lignes de force de la notion-clé dominant toute discussion pertinente à mon propos, celle de mémoire.

La mémoire est un élément essentiel de l'identité collective et individuelle. Que «l'homme de l'avenir» doive être une machine, une tekhnê numérique, on peut le croire, le craindre, le nier. Toutefois, mieux vaut éviter les projections hasardeuses en nous demandant tout modestement: y a-t-il dans la mémoire humaine quelque chose d'essentiel qui résiste à la numérisation? On peut penser à la dialectique de la mémoire et de l'oubli, et c'est la voie que je suivrai. Ce n'est qu'en fin de parcours que j'introduis, sans les traiter à fond, les quelques problemata qui sèment tant d'inquiétudes: la machine numérique parviendra-t-elle à préserver, à sauvegarder, à cultiver cette capacité mémorielle de l'oubli, à générer des anamnèses, à marquer d'une temporalité spécifique les différents modes de la productivité mnésique?

\section{MNEMOSYNĖ}

Les anges, soutient Dante, n'ont pas besoin de mémoire, car leur entendement est ininterrompu. Les êtres humains, en revanche, pour connaître, doivent se souvenir de phantasmes formés physiquement. Il y aurait par conséquent pour Dante des phantasmata qui sont des empreintes physiques et une procédure de réactivation de ces phantasmata dans la remémoration. Conception passablement contemporaine, dirionsnous, naturaliste même. N'importe. La mémoire, il est 
vrai, est une notion-carrefour puisqu'on parle de la «mémoire génétique», de la "mémoire historique», de la «mémoire ethnique», de la "mémoire écrite», voire de la "mémoire des ordinateurs". L'histoire de la «mémoire» est complexe. À l'époque archaïque, Mnemosynè, Mémoire, est une déesse, mère des neuf Muses. Elle préside à la haute poésie: le poète est un possédé de Mémoire. Versifier, pour Homère, est se souvenir. Cette divinisation mystique, extatique, fait de la mémoire une sagesse même, sophia. La mémoire est placée au commencement, elle est la matrice où s'inventent tous les arts humains, où naissent toutes les fabriques de l'homme, y compris la fabrication des idées. Mémoire et invention sont assimilées dans ce qui serait appelé aujourd'hui la «créativité». Pour penser, pour créer, l'homme a besoin de la machine mnésique, instrument mental qui génère tous les produits de l'esprit (Carruthers, 2002a: 17).

Mais on assiste vite à une dépréciation, une laïcisation même de la mémoire. Les systèmes rhétoriques, comme celui de Cicéron, évacuent la Mémoire (tout comme la Prononciation) dans la "Seconde Rhétorique». Mémoire et Prononciation ne sont que des amendements des trois disciplines de la "Première Rhétorique», inventio, dispositio et elocutio. La mémoire devient même un objet de scepticisme de la part des rhétoriciens et les instructions formulées se réduisent à l'entraînement et à l'acquisition d'une technique mémorielle. Cette dépréciation est déjà explicite chez Platon, où la philosophie en tant qu'épistémè est déclarée indépendante de la mémoire. La laïcisation de la mémoire se poursuit chez Aristote, où la mémoire est détachée de l'histoire mythique et déjà définie comme une phase tardive du travail rhétorique.

De mythique, la mémoire devient psychologique ${ }^{1}$ : elle est définie dans Aristote par la triade temps/ image/perception. Fonction psychique mystérieuse quand même, puisque l'être humain, par la mémoire, peut actualiser des impressions ou des informations passées qu'il se représente comme passées (Le Goff, 1986: 105sqq.). Les philosophies de la mémoire ont promptement découvert que les possibilités de stockage de la mémoire sont bien dépendantes de l'utilisation du langage, que les séquences mémorielles se déroulent canoniquement comme des récits, que la mémoire est manipulée par la censure individuelle, par les intérêts de l'affect et du désir, par le contrôle social. Puisque l'événement ou l'état des choses qui constitue son motif est absent, la mémoire déborde aisément par ses fantaisies, voire ses déformations. Une certaine maîtrise de cette mémoire «sauvage» est exercée à partir du moment où elle est engloutie dans les livres. L'imprimerie révolutionne la mémoire: la mémoire y est progressivement extériorisée. La «mémoire écrite» dilate rapidement la mémoire individuelle et collective, de sorte que Yates (1966) a pu écrire que le livre est l'agonie de l'art de la mémoire. Il va de soi que la soi-disant culture textuelle est avant tout une mémoire écrite. Toutefois, il reste une culture mnésique manifestée dans le comportement, surtout discursif, qui échappe à la domination de l'écriture et de la textualisation.

Les philosophies de la mémoire sont toutes affectées par une extrême métaphorisation. La mémoire est thesaurus, trésor, magasin, chambre forte, et elle est également tabula, tablette, empreinte. Métaphore architecturale et métaphore scripturale qui se complètent convenablement. Platon, dans le Théétète, énonce que les souvenirs sont emmagasinés dans la mémoire comme des pigeons dans un colombier. Cet assemblage d'un inventaire a une organisation interne: la mémoire sans ordre serait comme une bibliothèque sans catalogue. C'est bien la structure qui prime les «lieux de mémoire». Et cette structuration dépend de la nécessité d'un tri, d'une sélection. On peut s'entraîner, par des mnémotechniques, à trier, à sélectionner, toujours en fonction du crible de la mémoire qui est l'organisation interne de "magasin» mnésique.

La psychologie aristotélicienne exploite à fond la métaphore de la mémoire comme jeu de tablettes de cire, tabula memoriæ que Platon introduit dans le Théétète et qu'il dit emprunter à Homère:

Suppose [...] qu'il y ait dans nos âmes une cire imprégnable: en l'un de nous, plus abondante, en l'autre moins; en celui-ci 
plus pure, en celui-là plus encrassée; et plus dure ou bien, chez d'aucuns, plus molle, ou, chez certains, réalisant une juste moyenne. [...] C'est un don, affirmerons-nous, de la mère des Muses, Mnémosyne: tout ce que nous désirons conserver en mémoire de ce que nous avons vu, entendu ou en nous-mêmes conçu, se vient, en cette cire que nous présentons accueillante aux sensations et conceptions, graver en relief comme marques d'anneaux que nous y imprimerions. Ce qui s'empreint, nous en aurions mémoire et science tant qu'en persiste l'image. Ce qui s'efface ou n'a pas réussi à s'empreindre, nous l'oublierions et ne le saurions point. (Platon, Théétète, 191, d-e)

La mémoire comme bloc de cire, où les souvenirs sont comme des «marques», des sceaux dans la cire sur le modèle d'une inscription, d'une incision. La psychologie de la mémoire d'Aristote, mais de Cicéron et d'Augustin également, reprend l'idée selon laquelle la surface enduite de cire est empreinte d'images, mais des images qui ne sont pas des copies, mais bien plutôt des dessins.

Le souvenir est une image mentale [phantasma], une apparence physiquement inscrite sur la partie du corps qui constitue la mémoire. Ce phantasme est l'aboutissement de tout un processus de perception sensorielle (de source visuelle, auditive, tactile ou olfactive). Comment est formée cette image mentale? Le mouvement qui se produit imprime comme une empreinte l'impression sensible, comme on dépose sa marque avec un sceau. (Aristote, De memoria et sensibilia, 450a, 30)

Ainsi la remémoration est considérée comme un processus de visualisation mentale ou cognitive.

Retenons également que cette remémoration est un véritable dépistage, investigatio. L'actualisation des «marques» de la mémoire est de nature heuristique, elle est une interprétation entraînant toute la rhétorique de l'invention et mobilisant la faculté créatrice de l'imagination.

\section{EMMAGASINAGE ET REMÉMORATION}

La problématique pertinente concernant la mémoire en psychologie contemporaine découle directement de l'acquis aristotélicien. Les questions sont de deux ordres. Le premier type de questions concerne la nature et l'emmagasinage des marques mnésiques, en particulier les souvenirs et les anamnèses. Comment concevoir la base physique de ces marques mentales? Faut-il supposer une affectation physique du tissu cérébral? Qu'est-ce que ces entités mentales représentent si elles sont des dessins plutôt que des copies? Si l'information encodée est aussi bien auditive, olfactive, tactile que visuelle, comment voir l'encodage de sensorialités si hétérogènes? Le second type de question concerne la remémoration. Qu'est-ce qui garantit et stimule la remémoration? Quelles sont les stratégies heuristiques de "dépistage» de ces marques mnésiques? Quel est le rôle de l'habitude, de la capacité d'association dans ce processus? On ne peut nier que la neuropsychologie cognitive de la mémoire s'implante dans ce questionnement originairement aristotélicien.

La psychologie de la mémoire chez Aristote est une psychologie somatique. Les marques mnésiques sont en fait des affections physiologiques de l'âme (Carruthers, 1990 et 1998). L'organe récepteur est «impressionné» par la marque mnésique, comme le suggère la métaphore du sceau dans la cire. La perception est une condition sine qua non de n'importe quel processus mnésique. Même les images oniriques ont une origine perceptive: il est vrai que les images mentales nous reviennent en rêve de façon spontanée et non comme l'effet d'une remémoration consciente, mais ceci ne met pas en question son origine perceptive. La faculté de perception ne fonctionne que par le travail des sens, mais elle est en même temps porteuse d'une charge émotionnelle. Alors comment déterminer la «marque mnésique»? Comme une image qui est plutôt une forme. La mémoire emmagasine les «ressemblances» des objets du monde, voire des événements, quand ils nous sont apparus et nous ont affectés. Ces «ressemblances» ne sont pas de simples réitérations mimétiques ou des "copies» du réel. La marque mnésique n'a pas la même forme sensible que les formes des objets dans le monde. Ce qui ne la réduit pas à une simple abstraction, un calcul, un algorithme ou un pur schéma. «Dessin», plutôt que 
«copie», puisque l'image mnésique en tant que forme sensible a sa composante émotionnelle. Il ne faut pas nier que la mémoire a une utilité intellectuelle décisive et qu'elle intervient dans la plupart des processus rationnels. On peut même dire que, dans un certain sens, chaque marque mnésique est une cogitation, un jugement. Et comme chaque souvenir ou anamnèse est une occasion personnelle, ils subissent des interférences émotionnelles et pathémiques: la crainte, le désir, l'inconfort «façonnent» la forme sensible qu'est la marque mnésique. C'est ainsi que la psychologie somatique de la mémoire génère une anthropologie, une pédagogie, une diététique même. Anthropologie des âges et des tempéraments (Aristote, De memoria et sensibilia, 453a, 20), par exemple, là où Aristote explique que la mémoire des jeunes et des vieillards ne fonctionne pas de manière optimale puisque leurs corps est en pleine mutation, ou que les tempéraments mélancoliques sont trop fluides pour bien retenir les marques mnésiques et pour contrôler et diriger l'acte de réminiscence. Une pédagogie donc, avec des entraînements ou des exercices mnémotechniques, une diététique même, avec des conseils bien pratiques. Le cerveau doit rester froid et humide, pas de surchauffe par conséquent, pas d'activités immodérées, y compris sexuelles: seules les joies tempérées et les plaisirs honnêtes favorisent l'entretien de la mémoire.

La remémoration a sa propre autonomie psychologique. La remémoration, par opposition à l'emmagasinage, est un processus actif qui met en œuvre des techniques bien éprouvées comme l'association. Aristote semble donner un certain privilège à l'association par lieux. On remémore mieux si l'on suit la chaîne des lieux (apo tupon): on parcourt la disposition spatiale des objets situés l'un à côté de l'autre, qu'ils soient voisins, semblables ou contraires (Aristote, De memoria et sensibilia, 415b, 18). Et le Stagyrite insiste sur le fait que les liaisons associatives les plus puissantes sont formées de consuetudine, par habitude. La remémoration, maillon décisif entre un état de connaissance et son activation, peut être déterminée comme habitus, mais une habitude qui est un savoir-faire, une sapientia, une intelligence pratique qui n'est pas sans effets éthiques.

\section{LES MODES MNÉSIQUES}

Reformulons ces deux types de questionnement comme les deux niveaux d'une analyse phénoménologique de la mémoire. Dans cette perspective, mémoire et remémoration ne peuvent être séparées notionnellement. Ainsi mémoire/ remémoration, ou "se souvenir", est un acte intentionnel dont la noèse et le noème sont inextricablement liés. On se souvient toujours d'un quelque chose "noématique», et ce quelque chose «revient» dans la mémoire par un processus noétique. La noèse, par conséquent, est la visée: comment se souvient-on? Le noème est la chose visée, les souvenirs, si l'on veut, «les souvenirs qui se ruent au seuil de la mémoire", comme disait Augustin, des contenus spécifiques, jamais totalement vides et informes, puisqu'ils sont toujours marqués par un mode de donation. Même si les souvenirs «se donnent» dans toute leur immédiateté et clarté, comme c'est fréquemment le cas, ils sont marqués par un degré de densité et d'affectivité. En effet, la phénoménologie de la mémoire ${ }^{2}$ montre comment le processus noétique de l'acte de se souvenir n'est pas conceptualisant du tout, et plus, que cet acte est dominé par une dense signifiance émotionnelle. Ainsi l'acte intentionnel de se souvenir est souvent accompagné par des états d'âme forts comme les sentiments de nostalgie, de mélancolie, de regret.

On a pu noter, en phénoménologie de la mémoire, la familiarité dans le rapport noético-noématique: même si le souvenir doit "revenir" puisqu'il se trouve à une certaine profondeur qui doit être pénétrée par la visée mémorielle, il est vécu comme appartenant à l'intériorité du sujet intentionnel. Cette visée n'est pas vraiment éclairée par la lumière de la conscience, mais bien plutôt, comme Freud le démontre dans L'Interprétation des rêves, soumise à la censure, au choix inconscient, ou, si l'on suit Breton, aux manipulations de l'imagination. C'est que la mémoire est radicalement corporelle, nous enseigne la 
phénoménologie: pas de mémoire sans mémoire corporelle. Cette mémoire corporelle n'est pas la mémoire de la perception $d u$ corps, mais une mémoire intéroceptive. Bergson et Merleau-Ponty ont ouvert cette voie en constatant que la "mémoire d'habitude" est essentiellement de nature corporelle. D'une certaine façon, la mémoire est toujours, au moins partiellement, anamnésique. Il est vrai que l'anamnèse est globale dans la conception de la mémoire involontaire chez Proust, mais on pourrait généraliser ce point de vue. Ainsi Bergson note que le corps «porte» une immanence active du passé et qu'il «informe» les actions corporelles actuelles d'une façon orientée et efficace. Se sentir dépaysé quand on n'est pas assis dans son fauteuil de toujours suggère que la mémoire est intrinsèque au corps, qu'elle "passe» à travers le corps. C'est ainsi que l'acte intentionnel de se souvenir n'est pas thymiquement neutre: on souffre ou on jouit en se souvenant, toute mémoire est érotisée, voire traumatisée par le sentiment du corps propre.

Corporelle, la mémoire est, en plus, pleinement temporelle. Le temps est une dimension de toutes les marques mnésiques contenues dans la mémoire. Il ne faut oublier qu'une marque mnésique est à la fois l'«image» présente d'une chose et d'un événement passé, d'une part, et l'«image» d'une expérience révolue, de l'autre. Et se remémorer une expérience en tant que révolue est se remémorer un certain éloignement temporel, une durée relative du temps. Et encore, il se révèle possible de distinguer des modes mnésiques selon leur temporalité spécifique ${ }^{3}$. Edward Casey subtilise ainsi l'acte intentionnel de se souvenir en trois modes: le rappel («reminding»), la remémoration («reminiscing», "recollecting») et la reconnaissance («recognizing»). Peut-on affirmer que le temps passé qui «revient» dans l'acte de se souvenir est de plus en plus «éloigné» si l'on passe du rappel à la remémoration et de la remémoration à la reconnaissance? Le temps du rappel est court et le noème sémiotiquement spécifique et circonscrit. On se rappelle un rendez-vous, une date, un visage. Le temps de la remémoration, appelée souvent le souvenir, est «moyen": c'est le temps des autobiographies, des mémoires, des journaux. En général, la remémoration est marquée par le format extensible des récits. On se rappelle un fait, on se souvient d'un récit. Le temps de la reconnaissance est «long». On se rapproche de l'anamnèse complète puisque le contexte perceptuel actuel amène d'un passé, souvent très lointain, une même qualité sensorielle. Si le rappel concerne une anecdote factuelle et le souvenir un récit, la reconnaissance concernera bien plutôt une qualité sensorielle profondément enfouie dans un passé lointain.

\section{LESMOSYNE}

Mémoire est l'antidote d'Oubli. Le mythe est insistant: il ne faut pas boire au Léthé mais à la fontaine de Mémoire. Il est vrai, nos philosophies ne sont pas favorables à l'oubli. Freud: la psychanalyse sert à remplir les trous de la mémoire et constitue ainsi sa thérapie. Heidegger: le andenken ou la «pensée commémorative» est un effort pour transcender l'oubli de l'être. Nietzsche fait exception. Il faut cultiver l'oubli pour que les blessures de la vie soient éliminées. La mémoire rigide imposée par la loi est souvent anxieuse, il faut se libérer de cette culture de la mémoire pour vivre. Il est vrai, on comprend mieux la mémoire à travers sa déficience, l'oubli ${ }^{4}$. Et s'il y a, pour qu'il y ait communauté, devoir de mémoire, il y a certainement à pied égal devoir d'oubli. La mémoire et l'oubli sont solidaires, et pour ne pas perdre ni la mémoire ni la curiosité, il ne faut pas oublier d'oublier ${ }^{5}$. Il est trop facile d'énoncer que l'oubli est la perte du souvenir ou d'affirmer que la mémoire est la norme et l'oubli le refoulement, la dénégation. On sous-entend alors que l'oubli, même sous ses formes d'indifférence, de pardon ou de négligence, est du côté de la mort; la mémoire, dans le remords, l'obsession, la rancune, du côté de la vie. Mais il se fait que la vie et la mort, tout comme la mémoire et l'oubli, ne se définissent que l'une par rapport à l'autre. La mort, on n'en doute pas, est l'horizon de toute vie individuelle, et elle est perçue, jusque dans le quotidien, comme constitutive du sentiment de la vie. 
Ainsi l'oubli n'est pas la perte de la mémoire mais une composante essentielle de la mémoire elle-même. La mémoire est façonnée par l'oubli comme les rivages par la mer. Il faut reconnaître que l'oubli est nécessaire à l'individu comme à la société. Et si on fait l'éloge de l'oubli, ce n'est pas pour ignorer la mémoire, mais pour repérer le travail de l'oubli dans la mémoire. Le travail de l'oubli est un travail de sélection: pour que la mémoire s'épanouisse, il faut que certains souvenirs soient éliminés. Ce qui n'est pas en soi une mauvaise chose. Oublier un souvenir n'est pas encore oublier un fait, un récit, une qualité sensible dans leur extériorité absolue et indépendante, mais bien plutôt un certain traitement, une certaine interprétation de ce fait, de ce récit, de cette qualité sensible. Dans un sens, oublier n'est pas seulement sélectionner, mais également rectifier en fonction d'une plus grande cohérence, d'une familiarité plus authentique.

Loubli, comme la mémoire, incarne sa temporalité. Si la mémoire a besoin de l'oubli, c'est aussi pour restructurer le temps: pour focaliser l'intentionnalité sur la saveur de l'instant du présent ou sur la joie de l'attente, il faut "oublier» d'autres dimensions temporelles. Et inversement, notre rapport au temps passe essentiellement par l'oubli. Depuis Augustin, et en bonne phénoménologie, on sait que les trois dispositifs à gérer le temps - la mémoire du passé, l'attention au présent, l'attente du futur - s'entrelacent. Aucune dimension du temps ne peut se penser en faisant abstraction des autres. C'est ainsi que la tension entre mémoire et attente caractérise le présent, dans la mesure où il organise le passage d'un avant à un après. Marc Augé distingue, à partir de cette constatation, trois «figures" ou formes de l'oubli, les «trois filles de l'oubli». Le retour est la première forme de l'oubli. L'ambition première du retour est de retrouver un passé perdu, ce qui nécessite l'oubli du présent et de tout le laps du temps qui se confond avec lui. Et c'est par l'oubli du présent que le retour est en état de rétablir une continuité avec le passé le plus ancien. Le suspens est la seconde forme de l'oubli. Son ambition est de vivre le temps du présent en le coupant, au moins provisoirement, du passé et du futur. Le suspens consiste en une esthétisation de l'instant présent, passablement artificielle, où les dimensions du passé et du futur sont mises entre parenthèses ("oubliées») en fonction d'une hypostase existentielle du maintenant. Le (re)commencement, troisième forme de l'oubli, a comme ambition de créer les conditions d'une naissance ou d'une renaissance, d'une ouverture à tous les avenirs possibles. L'oubli, dans ce cas, consiste dans la réduction des dimensions du passé et du présent. Mythiquement, c'est le temps du départ, du voyage et de ses oublis.

On retiendra de cet excursus phénoménologique essentiellement trois propositions: l'immanence corporelle de la mémoire, la variété des modes mnésiques (rappel, remémoration, reconnaissance) et la force vive de l'oubli dans la constitution de la mémoire. Avant de poser adéquatement le problème d'une éventuelle numérisation de la culture mnésique, il convient d'ajouter à cet acquis phénoménologique quelques éléments d'une sémio-épistémologie de la mémoire.

\section{TRACE ET ARCHIVE:}

LE TRAVAIL SÉMIOTIQUE DE LA MÉMOIRE

Comment sémiotiser ces éléments de la psychologie post-aristotélicienne et de la phénoménologie husserlienne de la mémoire? Par «sémiotisation", on ne comprend pas plus que la constitution d'un cadre conceptuel «interdisciplinaire» combinant ces acquis psychologique et phénoménologique. Cette sémiotisation se construit à partir des métaphores inévitables de la trace et de l'archive. Traduisons "emmagasinage" par mise en place de traces et «remémoration" par relecture de ces traces. La trace est une incision. Elle est déconnectée de tout récit possible ou crédible, elle manque toute souplesse d'interprétation imaginative, elle n'évolue pas, elle s'est affranchie de l'histoire événementielle. Et pourtant, elle n'est pas purement spatiale - marque immobile et inamovible - mais spatio-temporelle. Une trace est aussi un tracé: il faut parcourir les souvenirs et les anamnèses. Tracé de l'écriture, comme le suggérait 
déjà Socrate dans le Philèbe: «La mémoire en sa rencontre avec les sensations et les affections (pathèmata) que provoque cette sensation, me semblent alors écrire (graphein) en nos âmes des discours» (38a39c). On l'a vu, la trace mnésique ${ }^{6}$, dans la conception «laïque» de la mémoire, est considérée comme une empreinte corporelle, cérébrale, corticale, empreinte matérielle, substrat physiologique établi par une causalité externe. Soyons conscient de ce glissement sémantique: de la trace en l'âme vers la trace dans le corps, de l'impression-affection vers l'empreinte. En plus, la notion de "trace» subit un autre déplacement. $\mathrm{Ne}$ dit-on pas que l'historien travaille sur les traces des cultures et des civilisations disparues, ces traces étant mi-matérielles mi-immatérielles? Matérielles en ce qu'elles sont écrites et témoignent ainsi d'une extériorité autonome. De la trace dans l'intimité de l'âme à travers la trace dans l'extériorité du corps, on atteint maintenant la trace dans une extériorité encore plus radicale, celle de graphisme, de l'écriture. Et on s'est approché ainsi de l'archive.

Cette extériorité radicale de l'archive est marquée par l'étymologie même du mot: archivum, archeion est un lieu de conservation pour les documents officiels. Les archives constituent, à côté des mythes, un des dispositifs les plus anciens du stockage de l'information 7 . Dans son extériorité quantitative, les archives sont de la mémoire inerte. Si inerte que l'on peut se demander si l'archivage, ce stockage méthodique de l'information, n'implique pas nécessairement une régression de la découverte intellectuelle, un ralentissement dans le processus d'objets nouveaux et de nouveaux savoirs. Quoi qu'il en soit, l'archivage est une passion et le goût de l'archive est risqué. «Plonger dans les archives»: archiver est une activité que l'on exprime en termes de plongée, d'immersion, de noyade. Distinguons d'emblée entre l'imprimé d'un texte et l'archive (Farge, 1989: 11-14). L'imprimé est un texte intentionnellement livré au lecteur. Destiné pour être lu, interprété et compris, le "texte imprimé», tel le livre littéraire ou scientifique, cherche à annoncer et créer une pensée, à modifier l'état du monde et des êtres humains. L'archive n'est rien de tel. L'extériorité quantitative de l'archive n'est sans doute pas sa caractérisation épistémologique la plus adéquate. On peut dire avec autant de droit que les archives livrent le non-dit, qu'elles forcent une brèche dans le tissu des jours quotidiens et sont tendues vers l'inattendu. Estce un sentiment naif que par le décodage de l'archive on déchire un voile, on traverse l'opacité d'un savoir et on accède à l'essentiel des êtres et des choses? Alors il ne serait plus question d'extériorité radicale, bien au contraire, puisque l'archivage agirait comme une mise à nu, comme la transgression de l'empiricité historique vers l'inaccessible essence.

L'épistémologie de l'archive, que Michel Foucault a développée dans quelques pages célèbres de L'Archéologie du savoir, indique ce pouvoir transgressif de l'archive. On n'a jamais mieux dit en quoi l'archive n'a rien d'extérieur et en quoi elle est plus proche de la «trace dans l'âme» que de la «trace graphique». L'élément de l'archive sollicite l'archéologie, non pas une archéologie des vestiges matériels, mais «une archéologie qui décrit les discours comme des pratiques spécifiées dans l'élément de l'archive» (Foucault, 1969: 172). Foucault explique cette conception de l'élément de l'archive de la façon suivante:

Dans l'épaisseur des pratiques discursives, des systèmes qui instaurent les énoncés comme des événements [...]. Ce sont ces systèmes d'énoncés [...] que je propose d'appeler archive. Parce terme, je n'entends pas la somme de tous les textes qu'une culture a gardés par-devers elle-même comme documents de son propre passé, comme témoignage de son identité maintenue; je n'entends pas non plus les institutions qui, dans une société donnée, permettent d'enregistrer et de conserver les discours dont on veut garder la mémoire [...]. [Les discours] naissent selon les régularités spécifiques. Que s'il y a des choses dites [...] il ne faut pas en demander la raison immédiate aux choses qui s'y trouvent dites ni aux hommes qui les ont dites, mais au système de la discursivité, aux possibilités et aux impossibilités énonciatives qu'il ménage. L'archive, c'est d'abord la loi de ce qui peut être dit, le système qui régit l'apparition des énoncés comme événements singuliers. Mais l'archive, c'est aussi ce qui fait que toutes les choses dites ne s'amassent pas indéfiniment dans une 
multitude amorphe [...], ne disparaissent pas au seul hasard d'accidents externes. [...] L'archive n'est pas ce qui recueille la poussière des énoncés redevenus inertes et permet le miracle éventuel de leur résurrection; c'est le système de son fonctionnement. (Ibid.: 169-171)

Et Foucault de poursuivre que l'archive est un niveau particulier entre la langue, le système de construction de phrases possibles, et le corpus, l'ensemble des paroles prononcées. L'archive est une pratique mais en même temps un système, «le système général de la formation et de la transformation des énoncés» ou encore «l'horizon général auquel appartiennent la description des formations discursives, l'analyse des positivités, le repérage du champ énonciatif (ibid.: 173). L'archive elle-même d'une civilisation, d'une culture, d'une société ne peut être décrite: elle est indescriptible, mais aussi incontournable. En tant que pratique systématique, l'archive n'est pas observable, ni "extériorisée» dans le corpus des discours actualisés, le «corps" physiologique et neuronal. Elle transgresse la corporéité pour fonctionner comme un «niveau de réalité» qui se situe entre le possible (la langue) et l'actuel (le corpus, la parole).

Cette épistémologie foucaldienne de l'archive peut sembler spéculative. Et pourtant, elle me semble extrêmement utile pour une sémiotique de l'activité mnésique. La mémoire n'est pas un trésor contenant le possible ou l'actuel, mais le réel archival: ce dont on se souvient n'est ni une reconstruction abstraite des possibilités, ni l'éparpillement des données actuelles et éphémères sans aucune consistance temporelle, mais les pratiques d'émergence de la sémiose. Ainsi la mémoire est une trace, mais une «trace dans l'âme» (non pas une «trace dans le corps»), ou une archive, mais une archive archéologique (non pas une archive quantitative et extérieure). Trace et archive se superposent si on se place au niveau sémioépistémologique du réel archival, transcendant ainsi la dichotomie langue/parole, le possible/l'actuel. C'est ainsi que l'emmagasinage est une mise en place des traces ou de l'archive mnésiques, tandis que la remémoration est la relecture de ces traces ou de cette archive. «Trace» et/ou «archive», nonobstant leur métaphoricité, ont un statut sémio-épistémologique similaire.

J'ai pu opposer vestige, trace et archive en guise d'introduction à ces réflexions. Les vestiges comme indices des cultures et de civilisations du passé sont, à première vue, globalement empiriques, extérieurs et quantifiables. Je n'ai offert aucune analyse du concept de vestige, mais il convient de rappeler que mêmes les vestiges, transposés par l'imagination et l'art (Piranesi), acquièrent une certaine intériorité: les vestiges en tant que ruines sont l'effet de la qualité d'une certaine intériorisation. Je ne poursuis pas cette piste en ce lieu et me limite à une homologation de la trace et de l'archive, de la mémoire purement mnésique et de la mémoire textuelle. On a souvent remarqué (Carruthers, 1990 et 1998) que les «textes», à notre époque, prennent exclusivement la forme de livres, mais que, dans une culture mémorielle, un «livre» n'est qu'un moyen parmi d'autres de se souvenir d'un «texte», d'apprivoiser sa mémoire. Mais on peut concevoir le texte également comme une trace dans la mémoire, une entité mnésique «tracée dans l'âme». Le mot latin textus vient de tisser, et on voit dans l'histoire de l'Antiquité et du Moyen Âge que la textualisation s'effectue par le biais de la memoria. Textus signifie «texture», l'œuvre de la memoria qui "tisse» le texte dans une dynamique productrice. Ainsi, la mémoire est un processus de textualisation, pratique systématique, tout comme l'archive dans l'épistémologie de Foucault. On est par conséquent enclin, en cette fin de parcours, d'abolir l'opposition conceptuelle entre le textuel (détaché de son incarnation livresque) et le mnésique, entre la mémoire de l'archive et la mémoire de la trace.

\section{QUESTIONS PRÉLIMINAIRES SUR LA NUMÉRISATION} DE LA CULTURE MÉMORIELLE

Muséalisation de la culture, domestication de la mémoire, est-ce l'effet produit par le médium technologique aux ressources inépuisables qu'est la numérisation? Perte définitive de la différence culturelle, perte des fruits imprévisibles d'un échange 
qualitatif, perte de douces passions comme la nostalgie, la mélancolie; voilà ce que l'on craint de cette revanche sournoise du mort sur le vivant qui s'annonce avec l'invasion de la numérisation. Trame qui tend à se confondre avec le territoire, comme la machine avec le corps et le software hyperréaliste avec la cogitation si humainement réaliste. Muséalisation extensive, domestication à capacité d'extension quasi illimitée, quelle est la perte irréparable?

Si l'on pense pouvoir capter le culturel dans le mémoriel, et que l'on transforme la problématique de l'héritage culturel en une problématique de sauvegarde du mémoriel, on tombe vite sur des distinctions faciles à faire entre la mémoire humaine et la mémoire électronique. Mémoire électronique, durée illimitée, aucune entropie et stabilité permanente; mémoire humaine, particulièrement instable et malléable. La mémoire électronique n'agit que sur l'ordre et selon le programme de l'homme, tandis que la mémoire humaine conserve un large secteur non informatisable. Et faut-il dire que la mémoire électronique n'est qu'une aide, un serviteur de la mémoire et de l'esprit humain?

Pour conclure, je préfère éviter ce genre de constatations plaintives et récapituler quelques propositions qui pourraient problématiser le projet général d'une numérisation de l'héritage culturel. Psychologiquement et phénoménologiquement, la numérisation peut-elle intégrer l'immanence corporelle de la mémoire, la variété des modes mnésiques et surtout la force vive de l'oubli dans la constitution de la mémoire? Sémiotiquement, la numérisation peut-elle sauvegarder, voire cultiver, la trace et l'archive - lieux de la mémoire-, ce réel entre le possible (le schéma, la déduction, l'algorithme) et le réalisé (le positif, l'empirique, l'histoire et les discours)? Sauvegarder et cultiver, c'est dire: simuler la vie dans le temps et non pas les ombres de la caverne de Platon ou les fantômes angoissants de la maison des morts.

\section{NOTES}

1. Cette psychologisation de la problématique concernant la mémoire mène jusqu'au livre bien connu de Luria (1968).

2. Les meilleures analyses phénoménologiques sont de la main de Casey (1987).

3. Casey (1987). Cette classification est reprise par Ricœur (2000).

4. On entend dire souvent: «Dis-moi ce que tu oublies, je te dirai qui tu es »; «Une mauvaise mémoire, ça s'entretient, ça se cultive »; «Une mauvaise mémoire, ça rajeunit»!

5. Voir quelques belles pages à ce propos dans Augé (1998).

6. Sur la notion de trace, voir Ricœur (2000: index).

7. Datées du début du II ${ }^{e}$ millénaire av. J.-C., les premières archives connues sont les tablettes découvertes dans les palais et les temples de Mésopotamie. Voir Traverses, nº 36.

\section{RÉFÉRENCES BIBLIOGRAPHIQUES}

ARISTOTE [1955]: De memoria et sensibilia, dans W. D. Ross, Aristoteles, Parva Naturalia, Oxford, Clarendon Press.

Augé, M. [1998]: Les Formes de l'oubli, Paris, Payot.

Bergson, H. [1929] : Matière et Mémoire: essai sur la relation du corps et de l'esprit, Paris, Alcan.

CARruthers, M. [(1998) 2002a]: Machina memorialis. Méditation,

rhétorique et fabrication des images au Moyen Âge, Paris, Gallimard; [(1990) 2002b]: Le Livre de la mémoire. Une étude de la mémoire dans la culture médiévale, Paris, Macula.

CASEY, E. [1987]: Remembering. A Phenomenological Study, Bloomington, Indiana U.P.

FARGE, A. [1989]: Le Goût de l'archive, Paris, Seuil.

FOUCAUlT, M. [1969]: L'Archéologie du savoir, Paris, Gallimard. IlliCH, I. [1991]: Du lisible au visible: la naissance du texte, Paris, Cerf. LE GOFF, J. [1986]: Histoire et Mémoire, Paris, Gallimard.

LuRIA, A.R. [1968]: The Mind of a Mnemonist. A little Book about a Vast Memory, Harmondsworth, Penguin Books.

Merleau-Ponty, M. [1949]: Phénoménologie de la perception, Paris, Gallimard.

Platon [2002]: Philèbe, trad., introd. et notes de J.-F. Pradeau, Paris, Flammarion;

— [1994] : Théétète, trad., introd. et notes de M. Narcy, Paris, Flammarion.

RiCCEUR, P. [2000] : La Mémoire, l'histoire, l'oubli, Paris, Seuil.

Traverses [1986]: "L'Archive ", no 36.

YATES, F.Y. [1966]: The Art of Memory, London, Ark Paperbacks. 